\title{
Curcumin for the prevention of progression in monoclonal gammopathy of undetermined significance: A word of caution (Review)
}

\author{
A.J.M. VERMORKEN ${ }^{1}$, J. ZHU ${ }^{1}$, W.J.M. VAN DE VEN ${ }^{1}$, Y. CUI ${ }^{3}$ and J.P. FRYNS ${ }^{2}$ \\ ${ }^{1}$ Laboratory for Molecular Oncology, Department of Human Genetics, University of Leuven; \\ ${ }^{2}$ Centre for Human Genetics, University Hospital Leuven, B-3000 Leuven, Belgium; ${ }^{3}$ National Engineering \\ Research Center for Miniaturized Detection Systems, Northwest University, Xi'an, Shaanxi 710069, P.R. China
}

Received December 16, 2009; Accepted January 19, 2010

DOI: 10.3892/etm_00000041

\begin{abstract}
A recent pilot study found that curcumin, in certain patients with monoclonal gammopathy of undetermined significance (MGUS), decreases the paraprotein load and the urinary $\mathrm{N}$-telopeptide of type 1 collagen bone turnover marker. While this result is encouraging, the easy availability of the food component turmeric, containing curcumin, may lead to intake by MGUS patients without medical supervision. Curcumin is generally considered safe. Nevertheless, it is known that curcumin inhibits interleukin-12 production in dendritic cells, thereby dampening the Th1 response. It is also well established that Th1 cells are protective against invading pathogens and tumors. The present study describes a case in which bronchitis developed upon turmeric intake for gastrointestinal complaints. While one case does not provide proof of curcumin toxicity, a thorough literature overview suggests that turmeric may have an immunosuppressive effect, notably in patients with a compromised immune system. A warning against the use of turmeric or curcumin without medical supervision in immunocompromised patients seems therefore very opportune. Patients with MGUS, in whom the levels of non-affected immunoglobulins are reduced, should be carefully monitored for toxicity when curcumin is administered.
\end{abstract}

\section{Contents}

1. Introduction

2. Turmeric toxicity in a mildly immunocompromised patient: a case study

Correspondence to: Dr Fons Vermorken, Laboratory for Molecular Oncology, Department of Human Genetics, University of Leuven, Herestraat 49/bus 602, B-3000 Leuven, Belgium

E-mail: fons.vermorken@med.kuleuven.be

Key words: monoclonal gammopathy of undetermined significance, dendritic cells, common variable immunodeficiency, curcumin, information society
3. Curcumin and the immune system

4. The role of dendritic cells in CVID

5. Curcumin and dendritic cells

6. Dendritic cells in patients with MGUS

7. Curcumin and the general public

\section{Introduction}

Curcumin, the major yellow pigment extracted from turmeric, has, over the last few years, been (re)discovered by modern science as a substance with great promise as a therapeutic agent. It is currently used in human clinical trials for a variety of serious conditions, including multiple myeloma, pancreatic cancer, myelodysplastic syndromes, colon cancer, psoriasis and Alzheimer's disease (1).

More recently, Golombick and Diamond (2) and Golombick et al (3) reported the results of pilot studies which indicated that curcumin, in some patients with monoclonal gammopathy of undetermined significance (MGUS), decreases the paraprotein load and the urinary $\mathrm{N}$-telopeptide of type 1 collagen bone turnover marker. MGUS indicates the presence of a monoclonal (M-) protein in individuals without evidence of multiple myeloma, Waldenström's macroglobulinemia, primary amyloidosis, or related disorders (4). The results with curcumin are promising, since an increasing paraprotein concentration is known to be a risk factor for the malignant progression of the above-mentioned diseases in these patients (5). The incidence of MGUS increases with age. The prevalence gradually increases from $1.7 \%$ in individuals between 50 and 59 years to $6.6 \%$ in individuals above 80 years of age (6). The average risk for malignant progression in an individual with MGUS is approximately $1.5 \%$ per year (7). MGUS is therefore a serious condition, as it leads to an increased risk of mortality, not only due to malignant transformation, but also due to coexisting clinical conditions (8). Nevertheless, many patients with MGUS never present symptoms and eventually succumb to unrelated causes. As a consequence, the medical profession has been very circumspect with treatment in MGUS. To date, only regular observation ('watchful waiting') with no treatment is recommended for these patients. This is based on the fear 
that treatment with, for example, alkylating agents may induce secondary leukaemia. Curcumin is believed not to pose this risk for various reasons, as outlined below.

Turmeric is widely consumed in Southeast Asia, and its use as a dietary spice and pigment is slowly growing worldwide. In India, in particular, it has also been used for centuries as a traditional medicinal product. Turmeric powder is easily available as a food component in Asia, but also in the Americas and Europe. Since it has been consumed for such a long time, the product is considered safe. Average intake in the adult Indian population is estimated at $2 \mathrm{~g}$ /day (containing up to $200 \mathrm{mg}$ curcumin). Phase I clinical trials indicate that even doses of up to $8 \mathrm{~g} /$ day of extracted curcumin (diferuloylmethane) provoke only minimal toxicity in healthy volunteers $(9,10)$. As a consequence, curcumin is believed not to be a risk factor for the induction of secondary leukaemia.

In a commentary on the above-mentioned study by Golombick et al (3), Rajkumar emphasized the crucial importance of developing new methods to prevent the malignant progression of MGUS (11). As mentioned, MGUS often does not present symptoms, while progression leads to incurable disease. A clear overview is presented of factors which, at the level of monoclonal cells as well their microenvironment, contribute to malignant progression and therefore constitute potential targets for primary and/or secondary prevention of such progression. Curcumin has been proposed as a potential agent for further trials, which ought to focus on cases at the highest risk of progression.

We find the first results with curcumin in MGUS very encouraging, and we agree with the points of view expressed by the above-mentioned authors and by Rajkumar. Further trials with the aim of delaying progression in MGUS are warranted. We do, however, feel the need to express a word of caution based on a case in which the use of moderate doses of turmeric induced some toxicity, possibly related to immunosuppression. The phenomenon was reproducible and can be understood on the basis of the scientific literature. Since immunosuppression is a risk factor for both the development and the progression of MGUS, we consider it useful to report on our findings.

\section{Turmeric toxicity in a mildly immunocompromised patient: a case study}

A 57-year-old male patient (from whom written informed consent was obtained) with mild hypogammaglobulinemia and a subnormal level of IgG caused by an IgG1 subclass deficiency (Table I) had been suffering from early childhood with otitis media, chronic rhinosinusitis and rarely bronchitis. The immune disorder was also present in other family members, sometimes combined with IgG3 deficiency. Throughout his life, the patient developed onychomycosis, plantar and flat warts, which were rather resistant to treatment. On two occasions, the patient reported that long-standing plantar warts, which had earlier been resistant to cryotherapy, X-ray irradiation, topical application with fluorouracil and injection with transfer factor, spontaneously cleared after intake of multivitamin tablets for several months at doses sometimes exceeding the recommended dietary allowance. The patient had no history of allergies. Chronic constipation in early life was followed by post infectious irritable bowel syndrome (IBS)
Table I. Characterization of the patient's IgG deficiency.

\begin{tabular}{lcc}
\hline Gammaglobulin g/l & Patient & Normal values \\
\hline Total gammaglobulins & 7.91 & $8.00-13.50$ \\
Total IgG & 7.18 & $7.51-15.60$ \\
IgG1 & 3.92 & $4.90-11.40$ \\
IgG2 & 2.29 & $1.50-6.40$ \\
IgG3 & 0.43 & $0.20-1.10$ \\
IgG4 & 0.13 & $0.08-1.40$ \\
\hline
\end{tabular}

from the age of 25 years. Prior treatment with salazopyrine had not improved the situation, and treatment with antibiotics had only a temporary effect. The encouraging pilot study by Holt et al (12) on the use of curcumin in inflammatory bowel disease led our patient to suggest the use of turmeric for the observed enteropathy. We proposed a dose of $1 \mathrm{~g}$, two times per day, of the food component. Holt et al prescribed 1-1.5 g/day, but used $99.5 \%$ pure curcumin (12). Nevertheless, our patient confirmed the positive results found by Holt et al; within weeks the complaints related to the enteropathy became significantly milder and the patient experienced a subjective improvement in quality of life. Positive results of curcumin for HIV-associated diarrhea were also described (13), further confirming the utility of curcumin for bowel pathology even in severely immunocompromised patients.

Within 6 weeks of the onset of treatment our patient developed mild bronchitis with chronic cough and wheezing. This was surprising since traditional Indian medicine recommends the use of turmeric for a number of health problems, including sinusitis (14). Turmeric treatment was discontinued, and was re-initiated several weeks later after the bronchitis had totally cleared. This time, the first signs of wheezing and bronchitis returned within 4 weeks. Turmeric was again discontinued for 3 weeks, and was subsequently re-initiated at a dose of $200 \mathrm{mg}$ two times per day. No wheezing or bronchitis developed, and the turmeric dose was still sufficient for a significant improvement of the intestinal problems. The patient has now used this treatment intermittently for almost 2 years with satisfaction and no toxicity.

Our patient has an IgG1 deficiency leading to mild IgG deficiency and borderline hypogammaglobulinemia. He has a proven weak and transient response to protein vaccines, and suffers from chronic rhinosinusitis and gastrointestinal problems. One in two patients with IgG1 deficiency develops ear, nose, throat and bronchial problems, while one in five patients develops gastrointestinal problems (15). The condition is therefore difficult to distinguish from a mild form of common variable immunodeficiency (CVID) (15). Notably, our patient's plantar warts cleared upon multivitamin intake. In a recent study (16) in which CVID was, as in the case of our patient, defined as a reduction in serum $\operatorname{IgG}(<7 \mathrm{~g} / \mathrm{l})$, recurrent bacterial airway infections and poor response to vaccinations, a vitamin B6 deficiency was noted in one out of the three patients. Vitamin B6 supplementation resulted in a significant increase in $\mathrm{CD}^{+} \mathrm{T}$ cells in these patients, while the concentration of serum immunoglobulins remained 
unchanged. Indeed, spontaneous resolution of plantar warts has been associated with an increase in $\mathrm{CD}^{+} \mathrm{T}$ cells $(17,18)$. CVID is a heterogeneous group of disorders characterized by hypogammaglobulinemia and a number of $\mathrm{T}$ cell defects that may be primary or secondary. In a number of patients, apparently including our patient, cellular immunity can be improved by supplementation with vitamin B6. It is tempting to speculate that the vitamin B6 deficiency is related to the gastrointestinal problems known to exist in a subgroup of patients with selective IgG1 deficiency (15).

The development of bronchitis after the use of turmeric or curcumin has, to the best of our knowledge, never been reported in the literature. Thus, it cannot be ruled out that our patient may have been more susceptible to turmeric toxicity than other patients due to his mildly compromised immune system and/or the related tendency to develop vitamin B6 deficiency. While one case does not provide proof for turmeric toxicity, a review of the literature is warranted in order to find or dismiss a possible rationale for an immunosuppressive effect of turmeric.

\section{Curcumin and the immune system}

A recent study in Wistar rats demonstrated that prolonged intraperitoneal curcumin injections did not impair the cytotoxic function of natural killer cells. Moreover, the generation of reactive oxygen species and nitric oxide from macrophages and levels of Th1 regulatory cytokines remained unaltered. On the basis of these results, the study concluded that curcumin is a safe pharmacological molecule for immune cells in vivo (19). The effect of curcumin on dendritic cells (DCs) was, however, not investigated. Nevertheless, it is well established that DCs are important for generating an immune response. After DCs are activated, they mature and migrate to the lymphoid tissue, where they prime $\mathrm{T}$ lymphocytes and stimulate a specific or adaptive response.

\section{The role of dendritic cells in CVID}

DCs play a key role in mucosal viral infections. Infection with respiratory viruses mobilizes immune cells, including both myeloid (m) and plasmacytoid (p)DCs, to the site of viral entry and promotes their replication in the respiratory tract. The increased number of pDCs in the nasal compartment significantly correlates with viral load, and is associated with a decrease in the number of pDCs in the blood. High numbers of both DC subsets are found in respiratory secretions weeks after the acute symptoms of the viral infection had resolved (20).

In patients with CVID, differentiation, maturation and the function of DCs are severely perturbed. DCs in CVID produce significantly lower amounts of interleukin-12 (IL-12) upon CD40 signaling as compared to controls. The expression of maturation markers, among them CD40 on CVID patient DCs, was also significantly lower than that of DCs from healthy donors. It has been found that, despite heterogeneous clinical presentation, CVID is generally associated with defective DC functions. Experiments with neutralizing anti-IL-10 monoclonal antibodies indicate that IL-10 may not be the only factor responsible for defective differentiation $(21,22)$.
Deficient IL-12 production by DCs is closely correlated to lymphocyte functions in vitro, and to the absolute numbers of CD4 T cells in peripheral blood. Although DCs in CVID patients appear morphologically similar to DCs in normal subjects, the lack of IL-12 production and defective antigen presentation demonstrate significant functional defects (23). On the other hand, it appears that CD40 ligand expression on stimulated T-helper lymphocytes in CVID patients is similar to that in normal controls (24). The above results, as well as a more recent study on selective deficits in blood dendritic cell subsets in CVID (25), point to an important role of defective differentiation and functioning of DCs in the pathogenesis of immunodeficiency in CVID.

\section{Curcumin and dendritic cells}

Almost 5 years ago, it was demonstrated that curcumin is a potent inhibitor of myeloid DC maturation in mice. Moreover, in vivo data showed that, although curcumin-treated DCs migrated to $\mathrm{T}$ cell areas of secondary lymphoid tissue, they failed to induce normal cell-mediated contact hypersensitivity. In particular, reduced IL-12 production observed after curcumin treatment indicated that curcumin induces the immature state of DCs. It was found that most of the effects of curcumin on the $\mathrm{T}$ cell-differentiating properties of DCs were the consequence of the inhibition of IL-12 production. Curcumin-treated DCs failed to induce a normal cell-mediated immune response. Therefore, it was proposed that curcumin could be used for inhibiting Th1 responses for therapeutical purposes (26). The activation of Th1 response plays a pivotal role in the induction of inflammation, such as in arthritis. On the other hand, Th1 cells are protective against invading pathogens and tumors. In other words, the anti-inflammatory effect obtained upon treatment of DCs with curcumin provoked, at the same time, an immunosuppressive effect.

A recent study (27) investigating the effects of curcumin on human DCs found that curcumin prevented DCs from responding to immunostimulants. DC-induced $\mathrm{CD}^{+} \mathrm{T}$ cell proliferation was prevented. The maturation markers were blocked, and the expression of cytokines and chemokines was inhibited. Migration was also reduced. It was concluded that curcumin dampens the Th1 response while promoting a Th2 permissive environment. The authors speculated that curcumin may be disrupting the antigen handling and presenting machinery of DCs. A potential therapeutic role for curcumin as an anti-inflammatory and immune suppressant was suggested (27).

Since a Th1 pattern of cytokine secretion, in which DC-derived IL-12 plays an essential role, has been associated with protective antitumor responses, dampening of the Th1 response by curcumin could be counterproductive in attempts to prevent malignant progression, at least in patients in whom DC maturation would be compromised.

\section{Dendritic cells in patients with MGUS}

In patients with MGUS and multiple myeloma (MM), which can result from malignant progression of MGUS, the existence of significant abnormalities in the distribution, phenotype and the pattern of secretion of inflammatory cytokines by different 
subsets of DCs from peripheral blood was demonstrated. Despite a reduced secretion of the inflammatory cytokines IL-1, $-6,-8$ and TNF in both MGUS and MM, the production of IL-12 after short-term in vitro culture was similar in patients with MGUS and controls (28). Whether curcumin inhibits IL-12 production from DCs in MGUS is a critical issue in view of the fact that immunosuppression is a known risk factor for the development of MGUS (11). Studies of familial clusters of Waldenstrom's macroglubulinemia, another lymphoproliferative disease often preceded by MGUS, have found an increased frequency of IgM MGUS compared to the general population, and have provided data suggesting that the phenotypic spectrum may also include hypoglobulinemia (29).

It is conceivable that immunosuppression by curcumin is only relevant in already immunocompromised patients with aberrantly functioning DCs, and not in healthy controls. In this context, it is well known that, in subgroups of MGUS patients, the non-affected immunoglobulins are reduced. These patients show, on average, a higher risk for progression (30). Whether, in these patients, maturation and IL-12 production of DCs would be perturbed by curcumin, and whether this leads to immunosuppression and the risk of progression of MGUS, are issues that must be addressed in future clinical trials.

\section{Curcumin and the general public}

Presently, in our developing 'information society', findings reported in the scientific literature, or at least parts thereof, are rapidly divulged to the general public. Moreover, companies now include 'natural substances' in their products for the health and cosmetic markets. For example, over the last few years the benefits of polyphenols have been presented in advertising campaigns for health, diet and beauty products, and the results of scientific studies have been used and quoted in this context. This practice could be positive, were it not that the increased public awareness concerning natural products has not countered the relatively widespread misconceptions regarding their toxicity. 'Natural' products are considered to pose no or only a minimal toxicity risk. In fact, natural products can, and do, induce significant toxicity when used by susceptible individuals, or when the dose is higher than that normally consumed at dietary levels. Since turmeric has been used for centuries, it is improbable that problems will arise in healthy individuals when curcumin is ingested at or around the quantities normally found in the diet. However, at significantly higher doses and/or in susceptible individuals, toxicity may occur. For example, the first case of complete atrioventricular block associated with turmeric intake was described and published online in November 2009 (31).

To date, scientific knowledge is insufficient for identifying all risk factors or risk groups. The identification of these will be the result of planned trials. We trust that the literature review and this illustrative case will alert the scientific community to the potential toxicity risk of curcumin in immunocompromised patients. We hope that the general public realizes that, at doses higher than those found in a regular diet, short- or long-term harmful side effects may occur, and that healthcare professionals will inform patients about the existence of such risks.

\section{Acknowledgements}

This study was supported by a grant from Fonds Wetenschappelijk Onderzoek Vlaanderen (G.0239.07N). The authors have also expressed their concern in a letter to the editor of Clinical Cancer Research.

\section{References}

1. Hatcher H, Planalp R, Cho J, Torti FM and Torti SV: Curcumin: from ancient medicine to current clinical trials. Review. Cell Mol Life Sci 65: 1631-1652, 2008.

2. Golombick T and Diamond TH: The potential role of curcumin (diferuloylmethane) in plasma cell dyscrasias/paraproteinemia. Biologics 2: 161-163, 2008.

3. Golombick T, Diamond TH, Badmaev V, Manoharan A and Ramakrishna R: The potential role of curcumin in patients with monoclonal gammopathy of undefined significance - its effect on paraproteinemia and the urinary $\mathrm{N}$-telopeptide of type I collagen bone turnover marker. Clin Cancer Res 15: 5917-5922, 2009.

4. Kyle RA: Monoclonal gammopathy of undetermined significance. Review. Blood Rev 8: 135-141, 1994.

5. Rosiñol L, Cibeira MT, Montoto S, Rozman M, Esteve J, Filella $\mathrm{X}$ and Bladé J: Monoclonal gammopathy of undetermined significance: predictors of malignant transformation and recognition of an evolving type characterized by a progressive increase in M protein size. Mayo Clin Proc 82: 428-434, 2007.

6. Kyle RA, Therneau TM, Rajkumar SV, Larson DR, Plevak MF, Offord JR, Dispenzieri A, Katzmann JA and Melton LJ III: Prevalence of monoclonal gammopathy of undetermined significance. N Engl J Med 354: 1362-1369, 2006.

7. Kyle RA, Rajkumar SV, Therneau TM, Larson DR, Plevak MF and Melton LJ III: Prognostic factors and predictors of outcome of immunoglobulin M monoclonal gammopathy of undetermined significance. Clin Lymphoma 5: 257-260, 2005.

8. Gregersen H, Ibsen J, Mellemkjoer L, Dahlerup J, Olsen J and Sørensen HT: Mortality and causes of death in patients with monoclonal gammopathy of undetermined significance. Br J Haematol 112: 353-357, 2001

9. Cheng AL, Hsu CH, Lin JK, Hsu MM, Ho YF, Shen TS, Ko JY, Lin JT, Lin BR, Ming-Shiang W, Yu HS, Jee SH, Chen GS, Chen TM, Chen CA, Lai MK, Pu YS, Pan MH, Wang YJ, Tsai CC and Hsieh CY: Phase I clinical trial of curcumin, a chemopreventive agent, in patients with high-risk or pre-malignant lesions. Anticancer Res 21: 2895-2900, 2001.

10. Sharma RA, Euden SA, Platton SL, Cooke DN, Shafayat A, Hewitt HR, Marczylo TH, Morgan B, Hemingway D, Plummer SM, Pirmohamed M, Gescher AJ and Steward WP: Phase I clinical trial of oral curcumin: biomarkers of systemic activity and compliance. Clin Cancer Res 10: 6847-6854, 2004.

11. Rajkumar SV: Prevention of progression in monoclonal gammopathy of undetermined significance. Clin Cancer Res 15: 5606-5608, 2009.

12. Holt PR, Katz S and Kirshoff R: Curcumin therapy in inflammatory bowel disease: a pilot study. Dig Dis Sci 50: 2191-2193, 2005.

13. Conteas CN, Panossian AM, Tran TT and Singh HM: Treatment of HIV-associated diarrhea with curcumin. Dig Dis Sci 54: 2188-2191, 2009.

14. Ammon HP and Wahl MA: Pharmacology of Curcuma longa. Planta Med 57: 1-7, 1991.

15. Lacombe C, Aucouturier P and Preud'homme JL: Selective IgG1 deficiency. Clin Immunol Immunopathol 84: 194-201, 1997.

16. Bierwirth J, Ulbricht KU, Schmidt RE and Witte T: Association of common variable immunodeficiency with vitamin B6 deficiency. Eur J Clin Nutr 62: 332-335, 2008.

17. Van der Steen P, van de Kerkhof P, der Kinderen D, van Vlijmen I and Happle R: Clinical and immunohistochemical responses of plantar warts to topical immunotherapy with diphenylcyclopropenone. J Dermatol 18: 330-333, 1991.

18. Lynn J, Knight AK, Kamoun M and Levinson AI: A 55-year-old man with hypogammaglobulinemia, lymphopenia and unrelenting cutaneous warts. J Allergy Clin Immunol 114: 409-414, 2004.

19. Varalakshmi CH, Ali AM, Pardhasaradhi BV, Srivastava RM, Singh S and Khar A: Immunomodulatory effects of curcumin: in vivo. Int Immunopharmacol 8: 688-700, 2008. 
20. Gill MA, Palucka AK, Barton T, Ghaffar F, Jafri H, Banchereau J and Ramilo O: Mobilization of plasmacytoid and myeloid dendritic cells to mucosal sites in children with respiratory syncytial virus and other viral respiratory infections. J Infect Dis 191: 1105-1115, 2005.

21. Bayry J, Lacroix-Desmazes S, Kazatchkine MD, Galicier L, Lepelletier Y, Webster D, Lévy Y, Eibl MM, Oksenhendler E, Hermine $\mathrm{O}$ and Kaveri SV: Common variable immunodeficiency is associated with defective functions of dendritic cells. Blood 104: 2441-2443, 2004.

22. Scott-Taylor TH, Green MR, Raeiszadeh M, Workman S and Webster AD: Defective maturation of dendritic cells in common variable immunodeficiency. Clin Exp Immunol 145: 420-427, 2006.

23. Cunningham-Rundles C and Radigan L: Deficient IL-12 and dendritic cell function in common variable immune deficiency. Clin Immunol 115: 147-153, 2005.

24. Ravanbakhsh M, Sarafnejad A, Aghamohammadi A, Kardar GA, Asgarian Omran H, Atarod L, Rezaei N, Shahrestani T, Hosseini M and Moin M: CD40 ligand expression on stimulated T-helper lymphocytes in patients with common variable immunodeficiency. Iran J Allergy Asthma Immunol 6: 129-135, 2007.

25. Yong PF, Workman S, Wahid F, Exley A, Webster AD and Ibrahim MA: Selective deficits in blood dendritic cell subsets in common variable immunodeficiency and X-linked agammaglobulinaemia but not specific polysaccharide antibody deficiency. Clin Immunol 127: 34-42, 2008.
26. Kim GY, Kim KH, Lee SH, Yoon MS, Lee HJ, Moon DO, Lee CM, Ahn SC, Park YC and Park YM: Curcumin inhibits immunostimulatory function of dendritic cells: MAPKs and translocation of NF-kappa B as potential targets. J Immunol 174: 8116-8124, 2005

27. Shirley SA, Montpetit AJ, Lockey RF and Mohapatra SS: Curcumin prevents human dendritic cell response to immune stimulants. Biochem Biophys Res Commun 374: 431-436, 2008.

28. Martín-Ayuso M, Almeida J, Pérez-Andrés M, Cuello R, Galende J, González-Fraile MI, Martín-Nuñez G, Ortega F, Rodríguez MJ, San Miguel JF and Orfao A: Peripheral blood dendritic cell subsets from patients with monoclonal gammopathies show an abnormal distribution and are functionally impaired. Oncologist 13: 82-92, 2008.

29. McMaster ML, Kristinsson SY, Turesson I, Björkholm M and Landgren O: Novel aspects pertaining to the relationship of Waldenström's macroglobulinemia, IgM monoclonal gammopathy of undetermined significance, polyclonal gammopathy and hypoglobulinemia. Clin Lymphoma Myeloma 9: 19-22, 2009.

30. Gregersen H, Mellemkjaer L, Ibsen JS, Dahlerup JF, Thomassen L and Sørensen HT: The impact of M-component type and immunoglobulin concentration on the risk of malignant transformation in patients with monoclonal gammopathy of undetermined significance. Haematologica 86: 1172-1179, 2001.

31. Lee SW, Nah SS, Byon JS, Ko HJ, Park SH, Lee SJ, Shin WY and Jin DK: Transient complete atrioventricular block associated with curcumin intake. Int J Cardiol: Nov. 3, 2009 (Epub ahead of print). 